\title{
Assessment of patients affected by rheumatoid arthritis eligible for biotechnological agents and evaluation of their healthcare resource utilization and related costs
}

\author{
L. Degli Esposti ${ }^{1}$, V. Perrone ${ }^{1}$, D. Sangiorgi ${ }^{1}$, L. Sinigaglia ${ }^{2}$ \\ ${ }^{1}$ Clicon S.r.l., Health Economics and Outcomes Research, Bologna, Italy; \\ ${ }^{2}$ Department of Rheumatology, Gaetano Pini Institute, Milan, Italy
}

\begin{abstract}
SUMMARY
Objective. To provide estimates of patients with rheumatoid arthritis (RA) eligible for biotechnological therapy and to evaluate their healthcare costs.

Method. An observational analysis was performed based on data-linkage between administrative databases of selected Italian Regional/Local healthcare departments. Data were then re-proportioned to the Italian population. Patients with RA diagnosis defined by discharge diagnosis and/or exemption code during 01/01/2013$31 / 12 / 2017$ were included. The criteria applied to evaluate the elegibility for biotechnological therapy were:

1) methotrexate (MTX)-treatment failure $\geq 6$ months and start of a different conventional-synthetic diseasemodifying antirheumatic drugs (csDMARD);

2) corticosteroid $\geq 6$ months with dosage $\geq 7.5 \mathrm{mg} / \mathrm{die}$;

3) MTX-contraindication (therapy or hospitalization for renal damage/interstizial lung disease/hepatic failure). Mean annual costs per patient included drugs, hospitalizations, outpatient services.

Results. Data re-proportioned to the Italian population estimated 318,328 RA patients: 43,361 with, 274,967 without biotechnological agents. Among the latter, 26,487(9.6\%) patients met $\geq 1$ criteria applied for eligibility: 1,896 had MTX-treatment failure and started another csDMARD; 15,833 received corticosteroid $\geq 7.5 \mathrm{mg} / \mathrm{die}$; 7,788 had MTX-contraindication. Regarding patients fulfilling two criteria, 107 had MTX-treatment failure followed by another csDMARDs and corticosteroid $\geq 7.5 \mathrm{mg} / \mathrm{die}, 53$ were treated with another csDMARDs after MTX-treatment failure and also presented MTX-contraindication, $810 \mathrm{had}$ corticosteroid $\geq 7.5 \mathrm{mg} / \mathrm{die}$ and MTX-contraindication. Mean total annual costs for patients estimated eligible for biotechnological therapy was $€ 3,132$, of which $€ 177$ related to drugs indicated for RA and $€ 2,955$ related to other direct costs.

Conclusions. According to our estimates, around 10\% RA patients not currently treated with biotechnological agents are eligible for such therapies, highlighting a trend of under-use in clinical practice for RA management.
\end{abstract}

Key words: Biotechnological therapy, real-world, rheumatoid arthritis, under-treatment.

Reumatismo, 2021; 73 (1): 5-14

\section{INTRODUCTION}

heumatoid arthritis (RA) is a chronic inflammatory systemic auto-immune disorder with a progressive course which affects primarily the synovial membrane of the joints, although extraarticular manifestations are often present as well (1). The Global Burden of Disease (GBD) 2010 study gave an estimation of the significant clinical burden of RA (2): of the 291 diseases analysed in the GBD study, RA was ranked as the $42^{\text {nd }}$ and $74^{\text {th }}$ highest contributor of global disability (calculated as years of life lived with disability) and overall burden (calculated as disability-adjusted life years), respectively (2).

$\mathrm{RA}$ is one of the most common chronic inflammatory conditions (3). Its global prevalence stands at around $0.24 \%-1 \%$ (2, $4)$. This estimate is likely to increase over the next decades because of the progressive ageing of the population. In Italy, RA prevalence is estimated approximately at
Corresponding author: Valentina Perrone Clicon Srl, Health, Economics and Outcomes Research Via Murri 9, 40137 Bologna, Italy E-mail: valentina.perrone@clicon.it 
$0.41 \%-0.48 \%$ (5). RA poses a great economic burden on patients, families and healthcare systems. These costs are set to increase further in the coming years particularly in countries like Italy, where the elderly population is expected to increase (6).

The clinical manifestations of RA are heterogeneous, and can lead to a limitation in the range of motion and impaired function of the affected joints (7). Currently, there is no cure for RA, and the available treatments aim to prevent structural progression of damage, control inflammation and rapidly reach a low disease activity state or remission (8). Therefore, an early initiation of RA therapy upon diagnosis is required to get control of the disease and achieve optimal outcomes. While the short-term use of glucocorticoids can offer a fast control of inflammation to reduce pain and swelling in case of acute flares, the mainstay of RA treatments relies on the Disease Modifying Anti-Rheumatic Drugs (DMARDs), that are able to control the inflammatory course for longer periods (8). DMARDs can be categorized in conventional synthetic (cs), biological (b) and targeted synthetic (ts) (9).

Accordingly to the latest European League Against Rheumatism (EULAR) guidelines (10), methotrexate (MTX), which is considered as the anchor drug for RA, or another csDMARD in case of MTX contraindications, is recommended as first line treatment. Therapy adjustments should then be made if a low disease activity/remission are not achieved after 6 months, or if no improvement is observed after 3 months from therapy initiation. Patients with associated poor prognostic factors that at this time-point do not respond to the first csDMARDs strategy, or patients that had at least 2 previous treatment failures with different csDMARD should receive also a bDMARD therapy (10).

Over the past decades, the increasing understanding of RA pathogenesis and the underlying mechanisms paved the way to the development of bDMARDs, that positively revolutionized RA management (11). bDMARDs showed indeed to be able to target pivotal proteins of the RA pathogenetic network, providing a valid therapeutic alternative for patient not responding to traditional therapies.

The increasing number of therapeutic options for RA demands more evidence from routine clinical practice to provide insight on the prescription patterns in the realworld rheumatology practice. Larger and more in-depth analyses are also needed to assess their therapeutic appropriateness.

In the Italian context, the analysis of administrative databases, depicting the realworld practice, can represent a useful tool for healthcare professionals to evaluate the implementation of evidence-based recommendations in clinical practice and reduce any potential deviations detected.

Although guidelines recommend to switch treatment in RA patients with an inadequate response to conventional drugs, international studies conducted in real-world settings highlighted that this may not always occur in everyday clinical practice $(12,13)$.

To investigate if this trend also applies to the Italian context, this study aims to estimate the number of patients with a diagnosis of RA who are eligible for a biotechnological therapy according to the EULAR recommendation (14), but are not actually treated with such drugs in an Italian realworld setting. Moreover, we conducted an economic analysis to estimate the direct healthcare costs for the Italian National Health Service (INHS) associated with RA patients treated with or eligible for biotechnological therapy.

\section{MATERIALS AND METHODS}

\section{Data source}

An observational retrospective cohort study was conducted by using the administrative databases of a pool of Italian Regional/Local Healthcare departments from Lombardy, Veneto and Apulia Regions. Data were then re-proportioned to the Italian population. To perform the analysis, the following databases were used: demographic database which contains patients' demographic data; pharmaceuticals database providing 
data on prescription as Anatomical-Therapeutic Chemical code (ATC), number of packages, number of units per package, and prescription date; hospitalization database which includes all hospitalization data as the discharge diagnosis codes classified according to the International Classification of Diseases, Ninth Revision, Clinical Modification (ICD-9-CM); exemption database, which provides exemption code and date of exemption.

In each database, the patient code was used to create an electronic link with all the other databases. To guarantee patients' privacy, an anonymous univocal numeric code was assigned to each subject included in the study, in full compliance with the European General Data Protection Regulation (GDPR) (2016/679). No identifiers related to patients were provided to the authors. All the results of the analyses were produced as aggregated summaries, which could not be associated with the individual patients, either directly or indirectly. An informed consent was not required for using encrypted retrospective information for research purposes. According to the Italian law (15), the local Ethics Committee of the departments involved in the study was notified of the study and approved it. The study followed the REporting of studies Conducted using Observational Routinelycollected health Data (RECORD) guidelines (16).

\section{Study population}

The analysis herein included all patients who were diagnosed with RA ascertained for the presence of hospitalization discharge diagnosis with ICD-9-CM code 714 and/or an active exemption code 006 from January $1^{\text {st }}, 2013$ to December $31^{\text {st }}$, 2017. The accuracy of the exemption code for RA identification was previously validated by Carrara et al. (17) and its use is in line with other studies following a similar methodology $(18,19)$. Patients not treated with biotechnological drugs were considered eligible for this therapy if they met at least one of the following criteria (Table I):

1) use of MTX for at least 6 months, then switch to a different csDMARD;
Table I - Criteria applied to identify RA patients eligible for biotechnological therapies.

\begin{tabular}{|l|l|}
\hline Name & Definition \\
\hline MTX failure & $\begin{array}{l}\text { use of MTX for at least } 6 \text { months, then switch } \\
\text { to a different csDMARD }\end{array}$ \\
\hline CS $\geq 7.5 \mathrm{mg} /$ die & $\begin{array}{l}\text { corticosteroid treatment for at least } 6 \text { months, } \\
\text { with a dose } \geq 7.5 \mathrm{mg} / \text { die }\end{array}$ \\
\hline MTX contraindication & $\begin{array}{l}\text { contraindication to MTX therapy, defined as patients } \\
\text { on therapy or hospitalized for renal damage, } \\
\text { interstizial lung disease or hepatic failure. }\end{array}$ \\
\hline Sensitivity analysis & \multicolumn{2}{|l}{} \\
\hline CS $\geq 5$ mg/die & $\begin{array}{l}\text { corticosteroid treatment for at least } 6 \text { months, } \\
\text { with a dose } \geq 5 \text { mg/die }\end{array}$ \\
\hline
\end{tabular}

cSDMARDs, conventional synthetic disease-modifying antirheumatic drugs; CS, corticosteroid; MTX, methotrexate; RA, rheumatoid arthritis.

2) corticosteroid treatment for at least 6 months at a dosage $\geq 7.5 \mathrm{mg} / \mathrm{die}$;

3) contraindication to MTX therapy, defined as patients in therapy or hospitalized for renal damage (ICD-9-CM codes: 580-589), interstitial lung disease (ICD-9-CM codes: 510-519) or hepatic failure (ICD-9-CM codes: 570573). The criteria applied were developed based on the guidelines in force during the analysis and were refined and validated by a pool of clinicians specialized in rheumatology.

\section{Study variables}

Patients were identified as treated with biotechnological therapies if they had a prescription for the following agents indicated for RA at the time of the analysis: abatacept (ATC code: L04AA24), adalimumab (ATC code: L04AB04), anakinra (ATC code: L04AC03), certolizumab (ATC code: L04AB05), etanercept (ATC code: L04AB01), golimumab (ATC code: L04AB06), infliximab (ATC code: L04AB02), rituximab (ATC code: L01XC02), tocilizumab (ATC code: L04AC07). csDMARDs analysed were: MTX (ATC code: L01BA01), leflunomide (ATC code: L04AA13), sulfasalazine (ATC code: A07EC01), cyclosporine (ATC code: L04AD01), azathioprine (ATC code: L04AX01), chloroquine (ATC code: P01BA01), hydroxychloroquine (ATC code: P01BA02). Corticosteroids analysed 
belonged to ATC code: H02. The mean daily dose was calculated as the sum of total dosage prescribed in a prescription divided by the days covered by such prescription.

\section{Sensitivity analysis}

Corticosteroids at a low-dose of $7.5 \mathrm{mg} /$ die were recommended in combination with csDMARDs as bridging therapy and should be gradually tapered within maximum 6 months. If they could not be completely stopped within this time frame, the DMARD therapy might have to be considered a failure. In the guidelines in force during the analysis (14), the term low-dose was changed in short-term, leaving to physicians the decision about the dose regimens. Therefore, we decided to conduct a sensitivity analysis considering a corticosteroid dosage $\geq 5 \mathrm{mg} /$ die instead of $\geq 7.5$ $\mathrm{mg} /$ die for at least 6 months (Table I).

\section{Direct healthcare costs analysis}

The mean annual direct healthcare costs per patient based on resource consumption were estimated in terms of drugs, hospitalizations, and outpatient services. The healthcare cost analysis was performed from the perspective of the Italian National Health Service (INHS), with costs reported in Euros $(€)$. Drug costs were evaluated using the INHS purchase price. Hospitalization costs were determined using Diag- nosis Related Group (DRG) tariffs, which represent the reimbursement levels by the INHS to healthcare providers.

Since in general costs are not normally distributed, Generalized Linear Models (GLM) were assessed in order to evaluate the correlation between costs and use of biotechnological therapies, considering age, male gender and biosimilar formulation as confounding variables. A gamma distribution and an identity link function (in order to retrieve non transformed costs) were applied; post estimation tests included residuals analysis and check for influential observations.

\section{Statistical analysis}

Categorical variables were expressed as frequencies and percentages. Percentages of patients eligible for biotechnological therapy are referred to proportion of patients with one or more criteria among patients not treated with this therapy. All statistical analyses were performed using STATA SE, version 12.0.

\section{RESULTS}

Data re-proportioned to the Italian population in $2017(\mathrm{~N}=60,589,445)$ estimated in this analysis a total of 318,328 patients affected by RA. According to the estimation, as showed in Figure 1, 43,361 of them

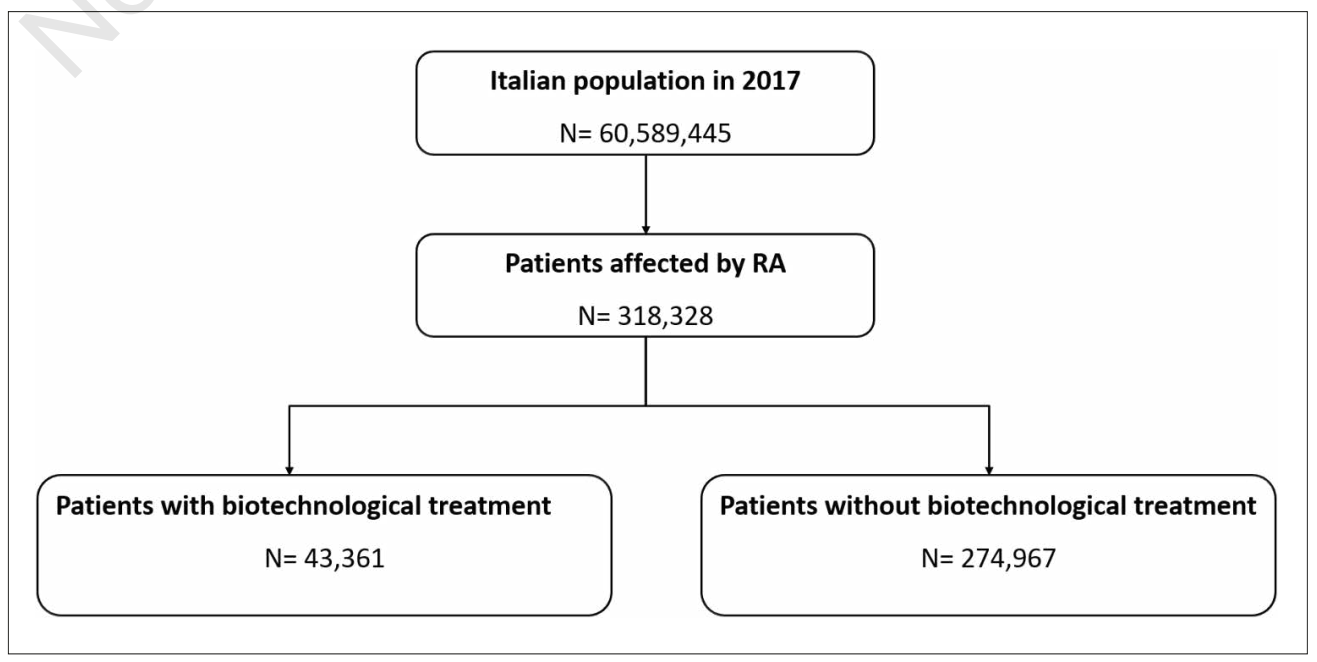

Figure 1 - Flowchart of the study population. Notes: data re-proportioned to the Italian population. RA, rheumatoid arthritis. 


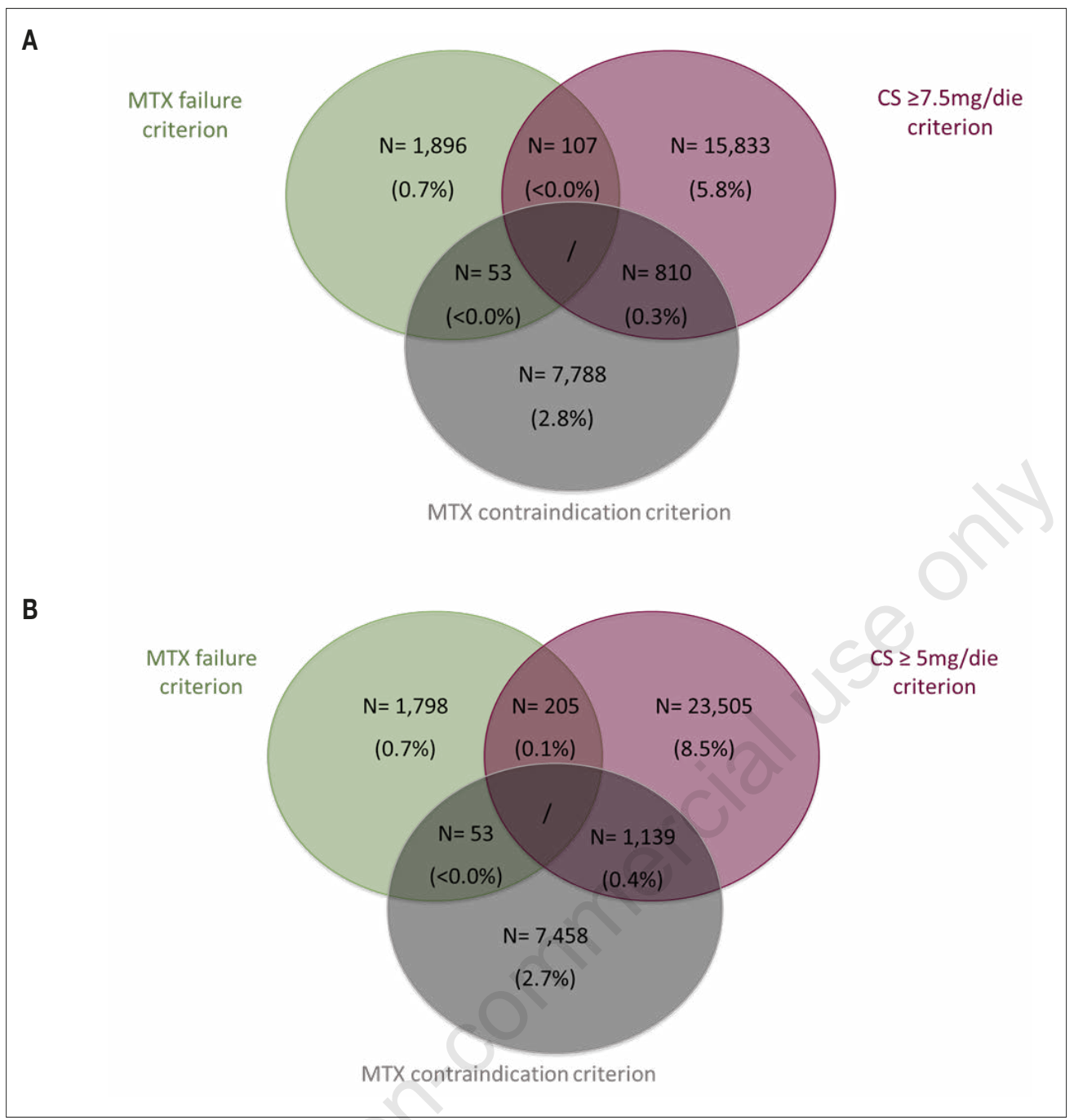

were treated with biotechnological treatments, while 274,967 were not prescribed with these drugs. Among the latter, 26,487 (9.6\%) patients were estimated to be eligible for biotechnological therapy, and their distribution according to the criteria applied is reported in Figure 2a. The demographic characteristics (re-proportioned) for patients with one criterion of eligibility and for patients receiving biotechnological agents are reported in Table II. As to patients who met one criterion of eligibility, $1,896(0.7 \%)$ (mean age $64.4,24.4 \%$ male) used MTX for at least 6 months, then switched to a different csDMARD (MTX failure criterion), 15,833 (5.8\%) (mean age $68.7,31.1 \%$ male) were on a corticosteroid $\geq 7.5 \mathrm{mg} /$ die for at least 6 months( $\mathrm{CS} \geq 7.5$ $\mathrm{mg} /$ die criterion) and 7,788 (2.8\%) (mean age $72.4,35.1 \%$ male) had MTX contraindication (MTX contraindication criterion). As to patients fulfilling two of the criteria applied, $107(<0.0 \%)$ used MTX for at least 6 months, then switched to a different csDMARD and were treated also with a corticosteroid $\geq 7.5 \mathrm{mg} /$ die for at least 6 months (MTX failure and CS $\geq 7.5 \mathrm{mg} / \mathrm{die}$ criteria), $53(<0.0 \%)$ were treated with another csDMARDs after MTX treatment for at least 6 months and also presented MTX contraindication (MTX failure and MTX contraindication criteria), $810(0.3 \%)$ were on a corticosteroid $\geq 7.5 \mathrm{mg} / \mathrm{die}$ for at least 6 months and had MTX contraindication (CS $\geq 7.5 \mathrm{mg} / \mathrm{die}$ and MTX contraindication criteria).
Figure 2

Distribution of patients eligible for biotechnological therapy according to criteria applied (a) and to sensitivity analysis (b). Notes: data reproportioned to the Italian population. Percentages referred to proportion of patients with 1 or more criteria among patients not treated with biotechnological therapy $(\mathrm{N}=274,967)$. CS, corticosteroid; MTX, methotrexate. 
A similar trend was also observed in the sensitivity analysis in which the criterion CS $\geq 7.5 \mathrm{mg} /$ die was replaced by a corticosteroid dosage $\geq 5 \mathrm{mg} /$ die for at least 6 months (CS $\geq 5 \mathrm{mg} /$ die criterion). In this analysis, eligible patients re-proportioned to Italian population were estimated to be 34,158 , representing $12.4 \%$ of patients without biotechnological treatment. Among them, as reported in Figure 3, 1,798 (0.7\%) patients met the MTX failure criterion, $23,505(8.5 \%)$ the $\mathrm{CS} \geq 5 \mathrm{mg} /$ die criterion and $7,458(2.7 \%)$ the MTX contraindication criterion (Figure 2b). In the same analysis, patients presenting two criteria were distributed as follow: $205(0.1 \%)$ satisfied
MTX failure and CS $\geq 5 \mathrm{mg}$ /die criteria; 53 $(<0.0 \%)$ MTX failure and MTX contraindication criteria; 1,139 ( $0.4 \%)$ CS $\geq 5$ mg/die and MTX contraindication criteria.

While patients receiving biotechnological therapy were mainly found in the age ranges between 60-69 (27.4\%) and 50$59(24.5 \%)$, patients with one eligibility criterion were mostly aged between 70 79 (28.2\% for MTX failure criterion and $32.3 \%$ for $\mathrm{CS} \geq 7.5 \mathrm{mg} /$ die criterion) or over 80 (35.8\% for MTX contraindication criterion) (Table II). Overall, among eligible patiens, the most common age range among women was $70-79(21.6 \%)$ followed by $80-89(12.8 \%)$ and $60-69(15.4 \%)$, while

Table II - Demographic characteristics of RA patients meeting one eligibility criterion for biotechnological therapy and RA patients treated with biotechnological therapies, as estimated in Italy.

\begin{tabular}{|l|c|c|c|c|}
\hline \multirow{2}{*}{} & \multicolumn{3}{|c|}{ Eligible patients } & \multirow{2}{*}{ Treated patients } \\
\cline { 2 - 3 } & MTX failure & CS $\geq 7.5 \mathrm{mg} /$ die & MTX contraindication & \\
\hline $\mathrm{N}$ & 1,896 & 15,833 & 7,788 & 73,361 \\
\hline Mean Age & 64.1 & 68.7 & 72.4 & 56.3 \\
\hline Male $\mathrm{n}(\%)$ & $463(24.4)$ & $4,931(31.1)$ & $2,732(35.1)$ & $9,852(22.7)$ \\
\hline \multicolumn{5}{|l|}{ Age range distribution n (\%) } \\
\hline$<40$ & $62(3.3)$ & $427(2.7)$ & $187(2.4)$ & $6,016(13.9)$ \\
\hline $40-49$ & $205(10.8)$ & $1,068(6.7)$ & $401(5.1)$ & $6,043(13.9)$ \\
\hline $50-59$ & $409(21.6)$ & $2,243(14.2)$ & $685(8.8)$ & $10,636(24.5)$ \\
\hline $60-69$ & $454(23.9)$ & $3,489(22.0)$ & $1,513(19.4)$ & $11,873(27.4)$ \\
\hline $70-79$ & $534(28.2)$ & $5,118(32.3)$ & $2,216(28.5)$ & $7,271(16.8)$ \\
\hline$\geq 80$ & $231(12.2)$ & $3,489(22.0)$ & $2,786(35.8)$ & $1,522(3.5)$ \\
\hline
\end{tabular}

CS, corticosteroid; MTX, methotrexate; RA, rheumatoid arthritis. Notes: data re-proportioned to the Italian population.

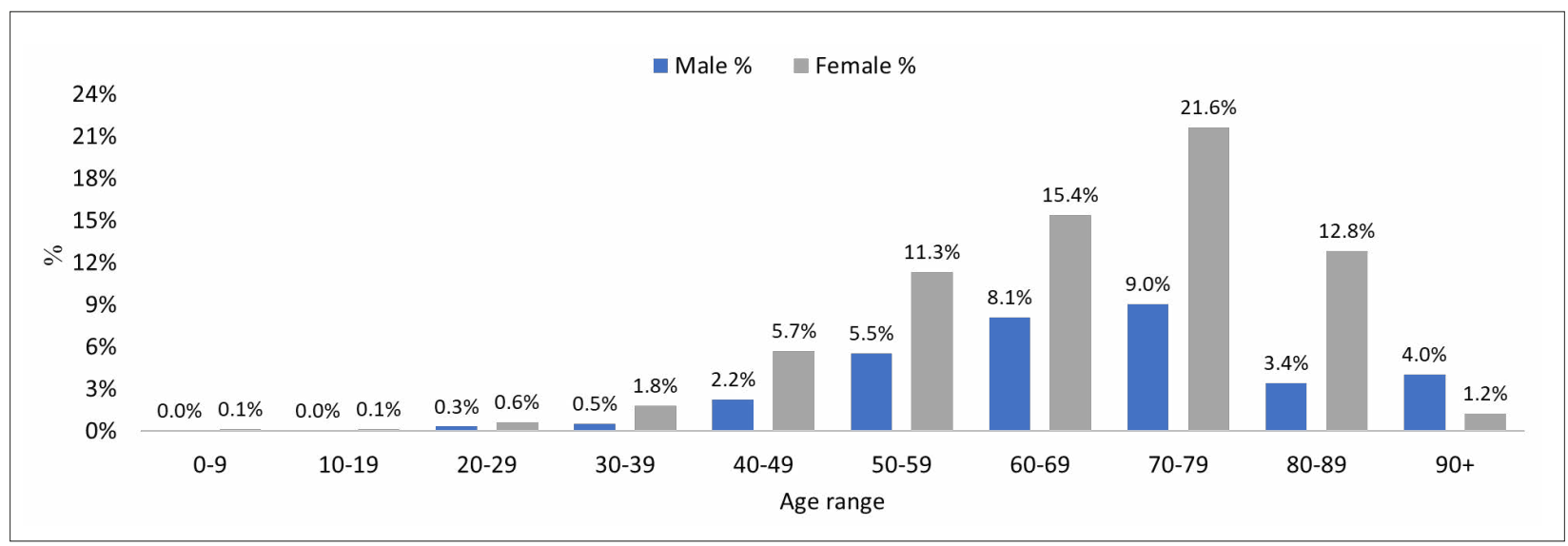

Figure 3 - Distribution of patients eligible for biotechnological therapy per gender and age. Notes: data re-proportioned to the Italian population. 


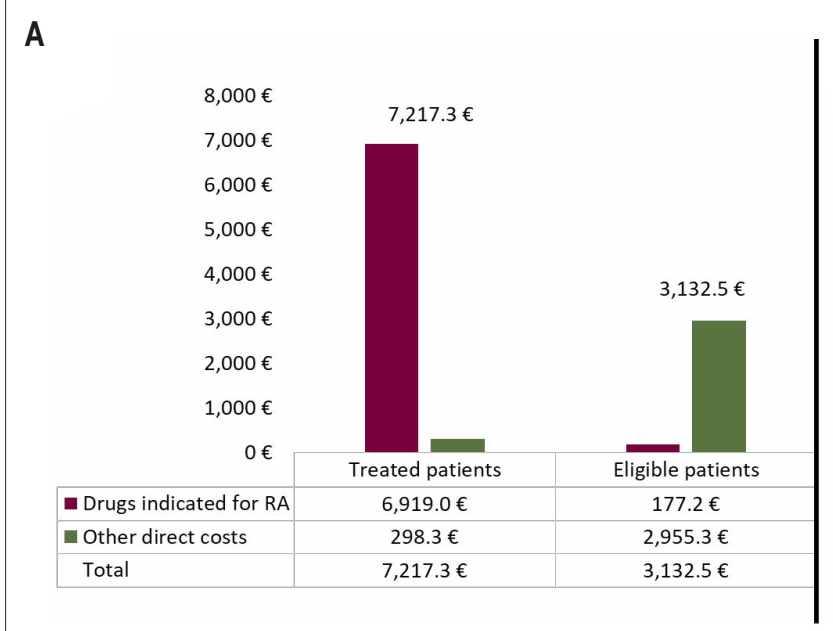

B

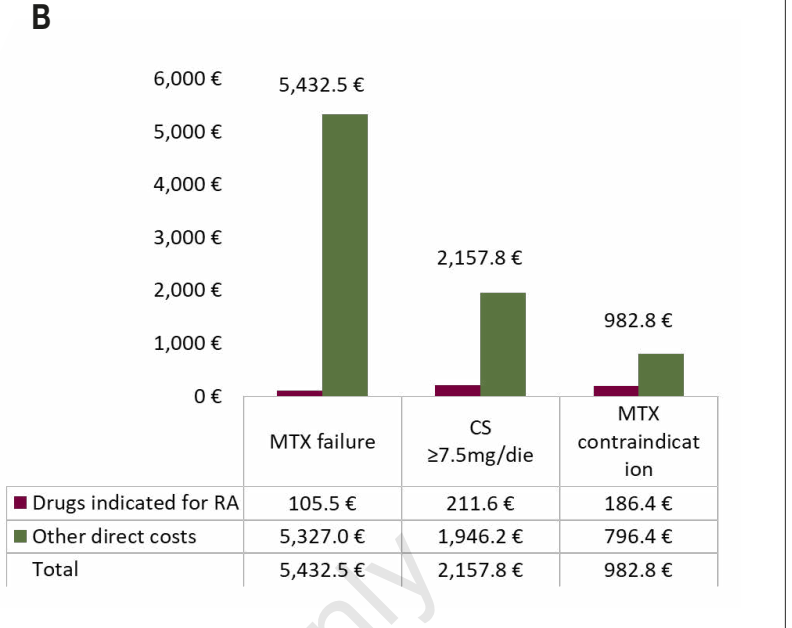

Figure 4 - Mean annual costs for patients treated with biotechnological therapy or eligible for biotechnological therapy (a) and stratified by elegibility criteria (b). Notes: data re-proportioned to the Italian population. Other costs include other drugs, hospitalizations, outpatient services. RA, rheumatoid arthritis.

among men the most common age ranges were $70-79,60-69$ and $50-59(9.0 \%, 8.1 \%$ and $5.5 \%$, respectively) (Figure 3 ).

The mean total annual healthcare cost (Figure 4a) for RA patients treated with biotechnological therapy was estimated to be $€ 7,217$, of which $€ 6,919$ for drugs indicated for RA and $€ 298$ for other direct costs. For patients eligible for such therapy, the estimated total annual expenditure was $€ 3$,132, of which $€ 177$ for drugs indicated for RA and $€ 2,955$ for other direct costs. Figure $4 \mathrm{~b}$ shows the mean annual costs for patients stratified by eligibility criteria, which reached $€ 5,432$ for patients with the MTX failure criterion, $€ 2,158$ for patients with the CS $\geq 7.5 \mathrm{mg} /$ die criterion and $€ 983$ for the MTX contraindication criterion. For patients who met each criterion, the other direct costs were the main determinant of the total expenditure.

Since eligible patients were generally older than those receiving biotechnological drugs, a GLM analysis was conducted taking into account potentially confounding variables. As reported in Table III, direct costs other than drugs indicated for RA were estimated to decrease significantly when using biotechnological drugs ( $€ 89.6$, $\mathrm{p}=0.027)$ and to increase with age (€ 19.3, $\mathrm{p}<0.001)$.
Table III - Generalized linear models for direct costs other than drugs indicated for RA.

\begin{tabular}{|l|c|c|c|c|}
\hline & $€$ & \multicolumn{2}{|c|}{$95 \% \mathbf{C l}$} & $\mathbf{p}$ value \\
\hline Use of biotechnological therapy & -89.6 & -169.0 & -10.2 & 0.027 \\
\hline Age & 19.3 & 17.1 & 21.5 & $<0.001$ \\
\hline Male gender & -53.3 & -132.8 & 26.2 & 0.189 \\
\hline Biosimilar formulation & 137.0 & -40.6 & 314.6 & 0.130 \\
\hline Intercept & 176.1 & 8.0 & 344.2 & 0.040 \\
\hline
\end{tabular}

\section{DISCUSSION}

Over the past 30 years, the availability of biotechnological treatments has transformed the management of RA. These drugs have proven to be highly effective in slowing disease progression and reducing RA symptoms, thus improving the quality of life of affected individuals (20-22). However, Italy is in the lowest positions in Europe in terms of access to biotechnological therapies $(23,24)$.

This study gave an estimation of RA patients at a National level who are eligible for biotechnological therapy, yet are not currently treated with these agents. To assess eligibility, 3 criteria were applied that reflected the recommendations reported in the EULAR guidelines concerning biotechnological drugs initiation in RA patients (14). Our estimation showed that 
out of 274,967 AR patients who were not treated with biotechnological therapies, from 26,487 up to 34,158 patients were eligible for such therapies, thus highlighting a trend of under-prescription in this patient population in Italy.

This tendency was also found in another Italian study, which investigated the underuse of biotechnological agents in clinical practice for several chronic diseases, RA included, for which these type of drugs are indicated (25). Based on a prevalence of 200,000 RA patients reported in literature (26), in that study a minimum of 43,000 and a maximum of 58,000 patients requiring biotechnological therapies were estimated, yet only 38,000 of them were on biotechnological treatment. Therefore, from a minimum of 5,000 to a maximum of 20,000 patients were calculated as being under treated with regard to these drugs. Over the last few years, several interpretations for this finding have been reported in the literature. The first reason could be related to the variability observed in terms of access to the prescription of biotechnological drugs. In the first social report on RA drafted by the Italian Society of Rheumatology (Società Italiana di Reumatologia, SIR) and the National Association of Rheumatic Patients (Associazione Nazionale Malati Reumatici, ANMAR) (27), patients who were more often prescribed biotechnological agents were treated in rheumatology centers, which are not evenly distributed across Italian territory, and only a small proportion were followed by general practitioners or rheumatologists. Moreover, the limited access to these therapies could be also a consequence of a delay in the diagnosis of RA. Indeed, the mean time from symptoms onset to confirmed RA diagnosis was estimated to be almost 1 year (27). There could be also economic reasons, since biotechnological agents have the drawback of costing more than the traditional therapy, which could limit their use. As a result, a higher number of patients on this therapy translates into a significant economic burden for the National Healthcare Systems, which should reconcile the need to contain health expenditure and avoid exceeding spending limits with the need to respect the right to health of all patients (28). In this regard, the advent of biosimilars could offer a lower-priced alternative (29). A further cause of biotechnological drugs under-use could be related to a therapeutic strategy different from the treat-to-target one claimed by international guidelines due to several reasons including age, presence of comorbidities and drugrelated risks (30).

The age distribution revealed that the population eligible for biotechnological therapy included mainly older peole. This is in line with other analyses reporting that younger patients are more likely to be prescribed biotechnological treatments than older ones, and there is a tendency of not prescribing these agents in the elderly (31-33). Nevertheless, our results showed a quite significant proportion of working-age patients being not prescribed this therapy: as a result under-treatment could contribute to worsen the burden placed on them by RA (34).

The economic analysis revealed that while total costs for patients treated with biotechnological drugs were mainly driven by drugs indicated for RA, those estimated for eligible patients were mostly affected by direct costs related to other drugs, hospitalizations and outpatient services. This trend emerged also when looking at patients meeting one of the eligibility criteria. The higher direct costs for patients eligible to the biotechnological therapy could be influenced by the older age observed in the eligibility cohort.

We acknowledge some limitations of the present study. Our cohort of patients reflected real clinical practice, but the results must be interpreted taking into account the limitations related to the observational nature of the study, which was based on data collected from administrative databases. The first one was the lack of clinical information related to the severity of RA in terms of disease activity, prognostic favourable/unfavorable factors and other potential confounders. Secondly, it was not possible to gain information from administrative databases on the reasons behind the underuse of biotechnological drugs, which could 
be related to clinical reasons or to different treatment strategies adopted. Moreover, the selected criteria could have over-estimated or under-estimated the number of RA patients eligible for biotechnological therapy in Italy. Finally, our study was based on estimates of the national population from a sample of Italian Regional/Local Healthcare departments which might be not fully representative of the Italian context.

\section{CONCLUSIONS}

In this analysis, data collected from administrative databases were re-proprtioned to the Italian population to provide an estimation at the national level of the number of patients affected by RA eligible for the biotechnological therapy. A characterization of these patients in terms of direct healthcare costs was also provided. Our results highlighted the under-treatment of a significant proportion (around 10\%-12\%) of RA patients who were considered eligible, yet actually did not receive any biotechnological treatments. Given the data source used in the study, we could not investigate the reasons underlying the trend of the underuse of these therapies in clinical practice for RA, which could be economic or organizational or due to prescribing decisions. The high direct healthcare costs for eligible patients could be mainly due to the older age observed in this cohort compared to patients who receive biotechnological therapies.

\section{Disclosure}

LS reports personal fees (invited speaker) from Amgen, personal fees (invited speaker) from Eli Lilly, personal fees (invited speaker) from UCB, personal fees (invited speaker) from Abbvie, personal fees (invited speaker) from Roche, personal fees (invited speaker) from BMS. LDE, VP, DS report no conflict of interest.

\section{REFERENCES}

1. Smolen JS, Aletaha D, McInnes IB. Rheumatoid arthritis. Lancet. 2016; 388: 2023-38.

2. Cross M, Smith E, Hoy D, et al. The global burden of rheumatoid arthritis: estimates from the Global Burden of Disease 2010 study. Ann Rheum Dis. 2014; 73: 1316-22.

3. Pahwa R, Singh A, Jialal I. Chronic Inflammation. In: StatPearls (Internet). Treasure Island (FL): StatPearls Publishing; 2020 (cited 2020 Feb 12). Available from: http://www.ncbi.nlm. nih.gov/books/NBK493173/

4. WHO. Chronic rheumatic conditions (Internet). WHO. (cited 2020 Feb 12). Available from: http://www.who.int/chp/topics/rheumatic/en/

5. Rossini M, Rossi E, Bernardi D, et al. Prevalence and incidence of rheumatoid arthritis in Italy. Rheumatol Int. 2014; 34: 659-64.

6. Benucci M, Rogai V, Atzeni F, et al. Costs associated with rheumatoid arthritis in Italy: past, present, and future. CEOR. 2016; 33.

7. Guo Q, Wang Y, Xu D, et al. Rheumatoid arthritis: pathological mechanisms and modern pharmacologic therapies. Bone Res (Internet). 2018 Apr 27 (cited 2019 Sep. 2); 6. Available from: https://www.ncbi.nlm.nih.gov/pmc/articles/PMC5920070/

8. Köhler BM, Günther J, Kaudewitz D, Lorenz H-M. Current therapeutic options in the treatment of rheumatoid arthritis. J Clin Med. 2019; 8 .

9. Silvagni E, Di Battista M, Bonifacio AF, et al. One year in review 2019: novelties in the treatment of rheumatoid arthritis. Clin Exp Rheumatol. 2019; 37: 519-34.

10. Smolen JS, Landewé RBM, Bijlsma JWJ, et al. EULAR recommendations for the management of rheumatoid arthritis with synthetic and biological disease-modifying antirheumatic drugs: 2019 update. Ann Rheum Dis. 2020; annrheumdis-2019-216655.

11. Favalli EG, Matucci-Cerinic M, Szekanecz Z. The Giants (biologicals) against the Pigmies (small molecules), pros and cons of two different approaches to the disease modifying treatment in rheumatoid arthritis. Autoimmun Rev. 2020; 19: 102421.

12. Harrold LR, Patel PA, Griffith J, et al. Assessing disease severity in bio-naïve patients with RA on treatment with csDMARDs: insights from the Corrona Registry. Clin Rheumatol. 2020; 39: 391-400.

13. Wei W, Knapp K, Wang L, et al. Treatment persistence and clinical outcomes of tumor necrosis factor inhibitor cycling or switching to a new mechanism of action therapy: realworld observational study of rheumatoid arthritis patients in the United States with Prior tumor necrosis factor inhibitor therapy. Adv Ther. 2017; 34: 1936-52.

14. Smolen JS, Landewé R, Bijlsma J, et al. EULAR recommendations for the management of rheumatoid arthritis with synthetic and biological disease-modifying antirheumatic drugs: 2016 update. Ann Rheum Dis. 2017; 76: $960-77$. 
15. Agenzia Italiana del Farmaco (AIFA). Guideline for the classification and conduction of the observational studies on medicines. (Internet) 2010. Available from: https://www. agenziafarmaco.gov.it/ricclin/sites/default/ files/files_wysiwyg/files/CIRCULARS/Circular\%2031st\%20May\%202010.pdf

16. Benchimol EI, Smeeth L, Guttmann A, et al. The REporting of studies Conducted using Observational Routinely-collected health Data (RECORD) Statement. PLoS Med. 2015; 12: e1001885.

17. Carrara G, Scirè CA, Zambon A, et al. A validation study of a new classification algorithm to identify rheumatoid arthritis using administrative health databases: case-control and cohort diagnostic accuracy studies. Results from the RECord linkage On Rheumatic Diseases study of the Italian Society for Rheumatology. BMJ Open. 2015; 5: e006029.

18. Silvagni E, Bortoluzzi A, Carrara G, et al. Comparative effectiveness of first-line biological monotherapy use in rheumatoid arthritis: a retrospective analysis of the RECord-linkage On Rheumatic Diseases study on health care administrative databases. BMJ Open. 2018; 8: e021447.

19. Iannazzo S, Furneri G, Demma F, et al. The burden of rheumatic diseases: an analysis of an italian administrative database. Rheumatol Ther. 2016; 3: 167-77.

20. Curtis JR, Singh JA. The use of biologics in rheumatoid arthritis: current and emerging paradigms of care. Clin Ther. 2011; 33: 679-707.

21. Nam JL, Winthrop KL, van Vollenhoven RF, et al. Current evidence for the management of rheumatoid arthritis with biological diseasemodifying antirheumatic drugs: a systematic literature review informing the EULAR recommendations for the management of RA. Ann Rheum Dis. 2010; 69: 976-86.

22. Chen Y-F, Jobanputra P, Barton P, et al. A systematic review of the effectiveness of adalimumab, etanercept and infliximab for the treatment of rheumatoid arthritis in adults and an economic evaluation of their cost-effectiveness. Health Technol Assess. 2006; 10: iii-iv, xi-xiii, 1-229.

23. Miltenburger $\mathrm{C}$, Günther $\mathrm{OH}$, Justo $\mathrm{N}$, et al. A Survey of barriers to treatment access in rheumatoid arthritis (Internet). 2009 (cited 2020 Feb. 14) p. 29. Available from: http://www. comparatorreports.se/RA\%20Barrier\%20Report_FINAL_050110.pdf

24. Federfarma. Farmaci biologici, in Italia poco utilizzati. I dati della Sir (Internet). (cited 2020 Feb 14). Available from: https://fed-
erfarma.it/Edicola/FiloDiretto/VediNotizia. aspx $? \mathrm{id}=19547$

25. Malattie autoimmuni: un esercito di 200mila pazienti senza accesso ai biologici (Internet). Italian Biosimilars Group. (cited 2020 Feb 13). Available from: http://www.italianbiosimilarsgroup.it/it/area-stampa/in-primopiano/158-malattie-autoimmuni-un-esercitodi-200mila-pazienti-senza-accesso-ai-biologici.html

26. Lucioni C, Mazzi S, Caporali R. Budget impact analysis of infliximab biosimilar: the Italian scenery. Global Region Health Technol Assess. 2015; 2: GRHTA.5000194.

27. D'Ambrosio G. Primo Rapporto Sociale sull'Artrite reumatoide in Italia SIR-ANMAR-Censis (Internet). Anmar Italia ONLUS. (cited 2020 Feb. 13). Available from: http://www.anmar-italia.it/news-doc/anmaristituzioni/202-primo-rapporto-sociale-sullartrite-reumatoide-in-italia-sir-anmar-censis

28. Fedele D, Marra A, Carletti R, Scanavacca P. Cost-effectiveness analysis of drugs biological in rheumatoid arthritis. Giorn Ital Farmacoecon Farmacoutil. 2015; 7: 44-52.

29. Gulácsi L, Brodszky V, Baji P, et al. Biosimilars for the management of rheumatoid arthritis: economic considerations. Exp Rev Clin Immunol. 2015; 11: 43-52.

30. Vermeer M, Kuper HH, Bernelot Moens HJ, et al. Adherence to a treat-to-target strategy in early rheumatoid arthritis: results of the DREAM remission induction cohort. Arthritis Res Ther. 2012; 14: R254.

31. Morsley K, Kilner T, Steuer A. Biologics prescribing for rheumatoid arthritis in older patients: a single-center retrospective crosssectional study. Rheumatol Ther. 2015; 2: 165-72.

32. Tatangelo M, Tomlinson G, Paterson JM, et al. Association of patient, prescriber, and region with the initiation of first prescription of biologic disease-modifying antirheumatic drug among older patients with rheumatoid arthritis and identical health insurance coverage. JAMA Netw Open. 2019; 2: e1917053e1917053.

33. Kalkan A, Husberg M, Hallert E, et al. Physician preferences and variations in prescription of biologic drugs for rheumatoid arthritis: a register-based study of 4,010 patients in Sweden. Arthritis Care Res. 2015; 67: 1679-85.

34. Taylor PC, Moore A, Vasilescu R, et al. A structured literature review of the burden of illness and unmet needs in patients with rheumatoid arthritis: a current perspective. Rheumatol Int. 2016; 36: 685-95. 